\title{
Mucoadhesive Nanoparticles: A Roadmap to Encounter the Challenge of Rapid Nasal Mucociliary Clearance
}

\author{
Chandrakantsing Vijaysing Pardeshi',*, Abhijeet Dattatraya Kulkarni', Raju Onkar Sonawane ${ }^{1}$, Veena \\ Shailendra Belgamwar ${ }^{2}$, Prashant Jagannath Chaudhari ${ }^{3}$, Sanjay Javerilal Surana ${ }^{4}$
}

${ }^{1}$ Department of Pharmaceutics, Industrial Pharmacy Laboratory, R. C. Patel Institute of Pharmaceutical Education and Research, Shirpur, Maharashtra, INDIA.

${ }^{2}$ Department of Pharmaceutical Sciences, R.T.M. Nagpur University, Nagpur, Maharashtra, INDIA.

${ }^{3}$ Department of Pharmaceutical Chemistry, R. C. Patel Institute of Pharmaceutical Education and Research, Shirpur, Maharashtra, INDIA.

${ }^{4}$ Department of Pharmacognosy, R. C. Patel Institute of Pharmaceutical Education and Research, Shirpur, Maharashtra, INDIA.

\begin{abstract}
The modulation of mucoadhesion at the nanoscale is a very challenging task before the formulation scientists. Mucoadhesive nanoparticles are endowed with distinct properties such as increased residence, intimate contact of mucoadhesive dosage form at the mucosal surface and reproducible drug absorption. Large surface area, porous endothelial membrane, high total blood flow, ready accessibility, rapid onset of action, low enzyme level compared to gastrointestinal tract and avoidance of hepatic first pass metabolism are few of the major reasons for preferred drug delivery across the nasal mucosal membrane. There is a limited systematic summarized literature is available which could outline the potential of mucoadhesive nanoparticles for intranasal administration and present review could be an excellent platform to fulfill the voids. The authors put very enthusiastic opinion that the nasal mucoadhesive nanocarriers would meet the criteria set by regulatory authorities and soon such formulations would be available to accomplish the healing desires of the community, provided a successful execution of extensive clinical research with encouraging outcomes.
\end{abstract}

Key words: Nanomedicine, Nanoparticles, Mucoadhesion, Intranasal, Drug delivery.

\section{INTRODUCTION}

Nanotechnology is a multidisciplinary field where Nano refers to the scale of objects measured in nanometers ( $\mathrm{nm}$, which is $10^{-9}$ of a meter). The dimensions of nanoparticles (NPs) are similar to biomolecules, such as proteins $(1-20 \mathrm{~nm})$, DNA ( diameter $2 \mathrm{~nm})$, virus $(\sim 20 \mathrm{~nm})$, cell surface receptors $(\sim 10 \mathrm{~nm})$ and hemoglobin $(\sim 5 \mathrm{~nm})$. Therefore, scientists with diverse backgrounds have clutched their attention to work with and understand properties of materials on a nano scale. ${ }^{1}$ Nanomedicine comes along one of the most important disciplines of nanotechnology and according to National Institute of Health (NIH), the term nanomedicine refers to highly specific medical intervention at the molecular scale for diagnosis, prevention and treatment of various diseases. Broadly, nanomedicines include nanopharmaceuticals, nanodiagnostics, nanotheranostics and nanobiomaterials. ${ }^{2-4}$

Last 20 years have witnessed an outburst in research on the development of novel drug delivery systems. Among them, the multiparticulate drug delivery systems have broad prospects in the pharmaceutical field, due to their superior outcomes vi\%: greater therapeutic efficacy and reduced dosing frequency. ${ }^{5,6}$ At the University of North Carolina, a chemistry Professor
Submission Date: 03-07-2018; Revision Date: 24-10-2018; Accepted Date: 29-12-2018

DOI: 10.5530/ijper.53.2s.45 Correspondence:

Prof. Chandrakantsing Vijaysing Pardeshi, Industrial Pharmacy Laboratory, Department of Pharmaceutics, R. C. Patel Institute of Pharmaceutical Education and Research, Shirpur, Maharashtra, INDIA

Phone: +919881414752 E-mail: chandrakantpardeshi11@gmail.com

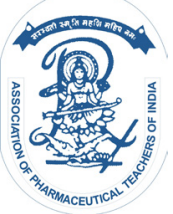

www.ijper.org 
Joseph De Simone once said about NPs that You want to deliver it where you want it, when you want it, without wasting $i t^{7}$ A very first report was published on the designing a specified drug delivery system to achieve selective targeting of drugs and was originated from the perception of Paul Ehrlich, who proposed a hypothesis of magic bullet concept. ${ }^{8}$

Drug delivery via nasal route has been practiced since ancient times for the treatment of local nasal disorders. Now, it has been recognized as a safe (being noninvasive) way to accomplish faster and higher levels of drug absorption. Large surface area, porous endothelial membrane, high total blood flow, ready accessibility, rapid onset of action, low enzyme level, avoidance of hepatic first pass metabolism and patient-friendly delivery using nasal administration devices are few of the major reasons for frequent intranasal drug delivery. ${ }^{9-12}$

Mucoadhesion (introduced in early 1980s) can be defined as the state in which two materials adhere to each other for extended periods of time with the help of interfacial forces and when one of these materials is biological in nature, the phenomenon is said to be bioadhesion. ${ }^{13}$ Longer and Robinson (1986) defined the term bioadhesion as the "attachment of a synthetic or natural macromolecule to mucus and/or epithelial surface". ${ }^{14,15}$ Mucoadhesion is gaining much attention today in formulation development of some mucoadhesive multiparticulate (micro or nano) drug delivery systems. Unlike single unit dosage forms, mucoadhesive multiparticulate dosage forms show some important merits viz: uniform distribution at the target site, more reproducible drug absorption and compact local irritation. ${ }^{16,17}$ To the best of authors' knowledge, there is no single review available illustrating the information on mucoadhesive nanocarriers for intranasal administration and present review could be a nice piece of paper for the researchers' engaged in developing the novel nanoparticulate dosage forms with mucoadhesion potential at the nasal mucosal surface. Looking at literature, the authors are confident about the future outcomes of the abundantly investigated mucoadhesive nanocarrier platforms particularly for intranasal administrations.

\section{Nanotechnology in Drug Delivery}

It all started way back in December 29, 1959, with a talk delivered by Dr. Richard Feynman (1918-1988), a physicist, wherein he presented the ideas and concepts behind nanoscience and nanotechnology in his presentation titled 'There's a Plenty of Room at the Bottom' at an international forum in the meeting of American Physical Society at the California Institute of Technology..$^{18-20}$
Over the last thirty years, nanomedicines have matured from proof-of-concept demonstrations in the lab into commercial products used in the clinic that are improving patient care as well as contributing to economic growth. The global market of nanomedicines was valued by the BCC Research firm (www.bccresearch.com) at \$209 billion in 2014 and is anticipated to expand to $\$ 412$ billion by 2019. The market share of nanomedicines represented $15 \%$ of the total pharmaceutical market in 2014 and is predicted to increase to $22 \%$ in $2019 .^{21,22}$

As evidenced by the significant nanopharmaceutical market, the application of nanotechnology in the field of medicine has the potential to improve the treatment of many diseases. Several nanomedicines have been approved by the US Food and Drug's Administration (FDA) and European Medicines Agency (EMA) for a variety of therapeutic indications. ${ }^{20-23}$

In history, nanocarriers were developed to encapsulate small molecules that suffer from low solubility, poor pharmacokinetic profile and high off-target toxicity. Lipid vesicles, later called liposomes, were among the first nanopharmaceuticals described in the 1960s. In 1980, targeting to specific regions of the body was demonstrated with $\mathrm{pH}$-sensitive liposomes. In 1987, the first long-circulating liposomes or stealth liposomes were described, introducing the concept of PEGylation. In 1995, doxorubicin (DOX) loaded PEGylated liposomes (named DOXIL in the USA and Caelyx in other countries) were approved for the treatment of AIDSassociated Kaposi's sarcoma. ${ }^{22}$

\section{Concept of mucoadhesion}

In the early 80's, Professor Joseph R. Robinson (19392006), a pharmacy researcher in the school of pharmacy at the university of Wisconsin (Madison, USA) pioneered the concept of mucoadhesion as a new strategy to prolong the residence time of variety of drug molecules on the mucosal surface of eye. ${ }^{24}$ This phenomenon has potential to optimize the controlled drug delivery in both ways vir. localized drug delivery (by spatial placement of drug-formulation within GI tract) and systemic drug delivery (by keeping the formulation in intimate contact with the tissues or cells at the absorption site). ${ }^{25}$

Mucoadhesion phenomenon has shown numerous pathbreaking advantages including (i) prolonged residence time enhances absorption, which results in an increase in the therapeutic efficacy of the drug, (ii) enormous blood supply and good blood flow rate causes rapid absorption of the drug, (iii) prevention of hepatic firstpass metabolism results in increase in drug bioavailability, (iv) avoidance of drug degradation due to acidic 
environment in the GI tract, (v) ease of drug administration, thereby improved patient compliance and (vi) faster onset of action due to mucosal surface. ${ }^{14,25,26}$

Mucoadhesion means simply an attachment of the drug-loaded carrier to the biological membrane. It is a complex phenomenon which involves several stages like wetting, adsorption and interpenetration of the polymer chains. A schematic diagram illustrating the mechanism of mucoadhesion is presented in Figure 1.27-29 The mucoadhesion takes place in two stages: (a) Contact stage: Intimate contact between a mucoadhesive NP formulation and a membrane (wetting or swelling phenomenon) and (b) Interactive stage (consolidation stage): Penetration of the mucoadhesive NPs into the tissue or into the surface of the mucous membrane. ${ }^{29}$ Numerous theories have been proposed to explain the above-mentioned mechanism of mucoadhesion. These theories of mucoadhesion along with their mechanism and key attributes are briefly summarized in Table $1 .{ }^{29}$

A mucoadhesive polymer is added to the pharmaceutical formulation in order to promote the adhesion of the formulation on the mucosal surface. An ideal mucoadhesive polymer must swell in the aqueous biological environment at the site of absorption, must interact with mucus or its components for adequate adhesion, must allow controlled release of active therapeutic when swelled and it must be excreted unaltered or be degraded to inactive or nontoxic oligomer. ${ }^{29,30}$ The classification of mucoadhesive polymers suitable for drug delivery applications is given in Figure 2.

\section{Intranasal drug delivery}

Traditionally, nasal route has been explored for delivery of drugs for the treatment of local nasal disorders. But, since the last few decades, nasal route has attracted wide attention as a reliable, safe (being noninvasive) and convenient route to accomplish faster and higher levels of drug absorption. The crucial reasons behind interest in nasal route are listed in introduction section of this manuscript. ${ }^{31-35}$

\section{Anatomy and physiology of nose}

Structurally, the nose is divided into two nasal cavities via a midline septum. The nasal cavity is about $12 \mathrm{~cm}$ long, the volume of each nasal cavity is $13 \mathrm{~mL}$ and has a total surface area of $\sim 150 \mathrm{~cm}^{2} .{ }^{9}$ Each cavity consists of three different regions namely: the vestibule $\left(0.6 \mathrm{~cm}^{2}\right)$, the olfactory region $\left(2-10 \mathrm{~cm}^{2}\right)$ and the respiratory region $\left(130 \mathrm{~cm}^{2}\right)$. The respiratory region contains three nasal turbinates, the superior, the middle and the inferior turbinate. These turbinates project from lateral wall of each half of nasal cavity and produces turbulent

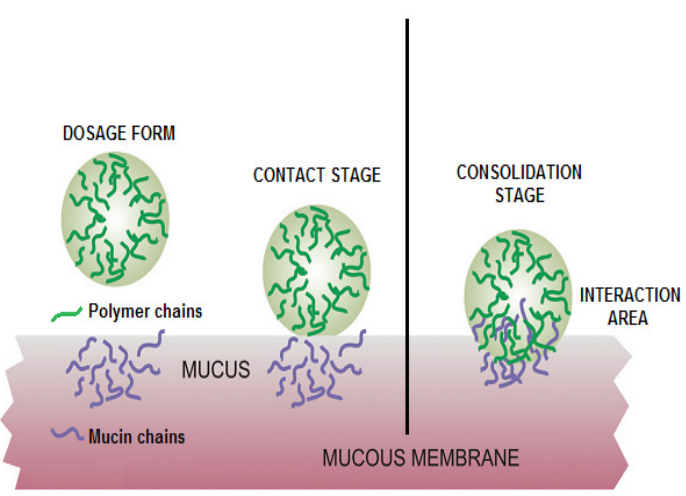

Figure 1: Mechanism of mucoadhesion.

The mucoadhesion takes place in two stages. (A) Contact stage: Intimate contact between a mucoadhesive formulation and a membrane (wetting or swelling phenomenon). (B) Interactive stage: Penetration of the bioadhesive into the tissue or into the surface of the mucous membrane (interpenetration). [Modified after 29].

\begin{tabular}{|c|c|}
\hline Theory & Mechanism and key attributes \\
\hline Wetting theory & $\begin{array}{ll}\checkmark & \text { Is primarily applied to liquid or low viscosity mucoadhesive systems } \\
\checkmark & \text { Affinity of the liquid to the mucosal surface inversely dependent on contact angle }\end{array}$ \\
\hline Adsorption theory & $\begin{array}{l}\checkmark \quad \text { Adhesive interaction among substrate surface depends upon the intermolecular forces such as } \\
\text { hydrogen bond, van der Waals' forces, etc. } \\
\checkmark \quad \text { Interaction across the interface occurs as a result of strong covalent bonding }\end{array}$ \\
\hline $\begin{array}{l}\text { Diffusion interlocking } \\
\text { theory }\end{array}$ & $\begin{array}{l}\checkmark \text { This theory proposes the time-dependent diffusion of mucoadhesive polymer chains into the } \\
\text { glycoprotein chain network of the mucus layer }\end{array}$ \\
\hline Electronic theory & $\begin{array}{l}\checkmark \quad \text { Electron transfer among surfaces resulting in attractive forces } \\
\checkmark \quad \text { Bonding occurs due to electron transfer between polymeric system and the mucus membrane } \\
\text { epithelium }\end{array}$ \\
\hline Fracture theory & $\begin{array}{l}\checkmark \text { This theory relates the force required for polymer detachment from the mucus to the strength of their } \\
\text { adhesive bond }\end{array}$ \\
\hline Mechanical theory & $\begin{array}{l}\checkmark \quad \text { This theory considers adhesion due to the filling of irregularities on a rough surface by a } \\
\text { mucoadhesive liquid } \\
\checkmark \quad \text { Such irregularity increases the interfacial area available for interactions }\end{array}$ \\
\hline
\end{tabular}




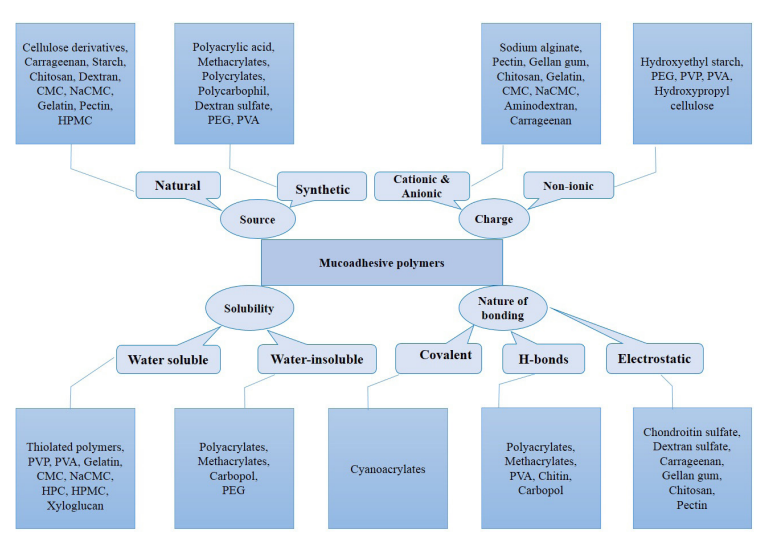

Figure 2: Classification of mucoadhesive polymers [Modified after [25]]

\begin{tabular}{|c|c|}
\hline \multicolumn{2}{|c|}{ Table 2: Composition of mucus. } \\
\hline Component & Percentage \\
\hline Water & $\sim 95 \%$ \\
\hline $\begin{array}{c}\text { Glycoproteins } \\
\text { (Sialomucin, fucomucin, sulfomucin) }\end{array}$ & $2 \%$ \\
\hline Salts (polyelectrolytes) & $1 \%$ \\
\hline $\begin{array}{c}\text { Proteins (Albumin, immunoglobulin) and } \\
\text { Enzymes (Lactoferrins, lysozymes) }\end{array}$ & $1 \%$ \\
\hline Lipids, cells, bacteria, cellular debris & $<1 \%$ \\
\hline
\end{tabular}

airflow through nasal passages, which ensures a better contact between inhaled air and mucosal surface. ${ }^{9,11}$

Functionally, the main functions of the nose are olfaction, regulation of humidity and temperature of inhaled air and removal of large particulates including microorganisms from the inhaled air. The nasal cavity plays an important protective role to filter, warm and humidify the inhaled air before it reaches the lower airways. It provides supply and conditioning of air to lungs. ${ }^{36-38}$

\section{Mucociliary clearance mechanism}

Any inhaled particle or microorganisms are trapped by the hairs of nasal vestibule or by mucus layer covering respiratory area of nasal cavity. Mucociliary clearance mechanism of mucus layer gradually carries such particulates to back of the throat, down the esophagus and further into gastrointestinal tract. Nasal mucosa also has metabolic capability of converting endogenous materials into compounds that are eliminated more readily. ${ }^{37,38}$

About $15-20 \%$ of respiratory cells are covered with a layer of mobile cilia (2-4 $\mu \mathrm{m}$ long, hair-like structures), which helps in propulsion of mucus towards the pharynx. Cilia are sensitive to temperature (optimally working at $35-40^{\circ} \mathrm{C}$ ) and their natural beat frequency drops below these temperatures. ${ }^{9}$
The composition of mucus is presented in Table 2. The goblet cell and the submucosal glands secrete about $20-40 \mathrm{ml}$ mucus per day, in human beings. The $\mathrm{pH}$ of the mucus varies from 5.5-6.5 in adults whereas 5-7 in infants. ${ }^{39-41}$ The mucus layer protects the underlying tissues from various environmental factors and the metabolic effects of enzymes. The mucus layer and hairs in the anterior nose filter $80 \%$ of particles larger than $12.5 \mu \mathrm{m}$ out from the inhaled air stream. ${ }^{42}$

The mucociliary clearance mechanism (MCC) is a very efficient defense mechanism in humans protecting the lungs against inhaled particulates, droplets and microorganisms. Mucus is present in two layers on epithelium in order to propagate mucociliary clearance. A 2-4 $\mu$ thick viscous mucus blanket called as 'gel layer' (Figure 3c), which floats on the 3-5 $\mu$ thick serous fluid layer called as 'sol layer' (Figure 3d). The viscous gel layer is moved along by the hook shaped cilia termini during energy dependent effective stroke phase of ciliary motion (Figure 3a). Cilia are up to $7 \mu$ in length when fully extended but can fold to half this length during recovery stroke (return beat). During recovery stroke, the hook terminus disengage from gel layer and moves immersed in the sol layer in opposite direction to the movement of gel layer (Figure 3b). The cilia beat with a frequency of 1000 strokes per min. These coordinated strokes of cilia result in movement of mucus in one direction only (Figure 3e) from anterior to posterior part of nasal cavity up to the nasopharynx. Therefore, particles applied on nasal respiratory mucosa will be transported on the mucus to the back of throat. The mucus flow rate is $5 \mathrm{~mm} / \mathrm{min}$ (with a range of $0.5-23.6 \mathrm{~mm} / \mathrm{min}$ ) and hence mucus layer is renewed every $15-20 \mathrm{~min}$. In humans, mucociliary flow can be measured by means of gamma scintigraphy or saccharine clearance test., ${ }^{9,37-40}$

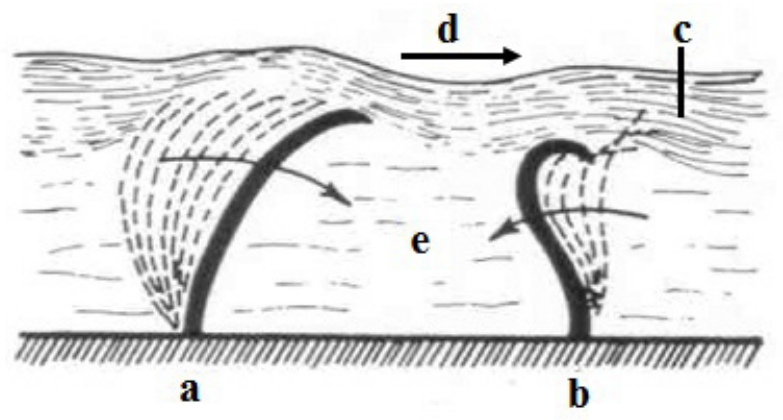

Figure 3: The relationship between ciliary motion and mucus layer composition that allows mucociliary clearance (a) Effective stroke, (b) Recovery stroke, (c) Gel layer, (d) Direction of gel layer movement and (e) Sol layer.

[Adapted from 9 with kind permission of the copyright holder, Elsevier, Amsterdam]. 
Nasal mucociliary clearance limits the residence time of intranasally administered formulation, thereby decreasing the extent of nasal drug absorption. Mucociliary clearance can be modulated by using specific components of the formulation vir: viscosity enhancers and mucoadhesive polymers. $^{39}$

\section{Mucoadhesive nanoparticles for intranasal administration}

This section deals with the various mucoadhesive NPs developed so far for intranasal administration. These nanocarriers are prepared by either of the following three ways vi: i) by using mucoadhesive polymers, ii) by modifying the nanocarrier formulation with mucoadhesive polymers or iii) by incorporating the nanocarrier formulation into the mucoadhesive polymer-based gel. The authors have categorized the nasal mucoadhesive nanocarrier formulations into three categories vir: lipid-based, polymer-based and protein-based nanocarriers for readers' convenience. The details of respective categories of NPs are detailed in Table 3.

Table 3: List of selected mucoadhesive nanoparticles investigated for intranasal drug delivery.

\begin{tabular}{|c|c|c|c|c|}
\hline Therapeutic payload & Mucoadhesive polymer & $\begin{array}{l}\text { Nannocarrier } \\
\text { formulation }\end{array}$ & Therapeutic applications & Reference \\
\hline \multicolumn{5}{|c|}{ LIPID-BASED NASAL MUCOADHESIVE NANOCARRIERS } \\
\hline BACE1 SIRNA & Chitosan & SLNs & Alzheimer's disease & 43 \\
\hline Carbamazepine & Pluronic F-127 (gel) & SLNs & Epileptic seizures and trigeminal neuralgia & 44 \\
\hline Ondansetron $\mathrm{HCl}$ & Delonix regia gum & NLCs & Chemotherapy-induced nausea and vomiting & 45 \\
\hline Ropinirole $\mathrm{HCl}$ & HPMC K15M & PLNs & Parkinson's disease & 46 \\
\hline Insulin & Chitosan & Liposomes & NM & 47 \\
\hline Acyclovir & PVP (gel) & Liposomes & HSV infections & 48 \\
\hline DNA & Glycol chitosan & Liposomes & Viral-specific immunization & 49 \\
\hline \multicolumn{5}{|c|}{ OIL-BASED NASAL MUCOADHESIVE NANOCARRIERS } \\
\hline Zolmitriptan & Polycarbophil & MEs & Migraine & 50 \\
\hline Sumatriptan & Polycarbophil & MEs & Migraine & 51 \\
\hline Clonazepam & Polycarbophil & MEs & Myoclonic seizures & 52 \\
\hline Tacrine & Carbopol $934 \mathrm{P}$ & MEs & Alzheimer's disease & 53 \\
\hline $\begin{array}{l}\text { Diazepam, Lorazepam } \\
\text { and Alprazolam }\end{array}$ & Polycarbophil & MEs & Insomnia & 54 \\
\hline Risperidone & Chitosan & NEs & Psychotic disorders & 55 \\
\hline Risperidone & Chitosan & NEs & Psychotic disorders & 56 \\
\hline Zaleplon & Carbopol 934 (gel) & NEs & Insomnia & 57 \\
\hline Curcumin & Chitosan & NEs & Cancer & 58 \\
\hline Ropinirole & Chitosan & NEs & Parkinson's disease & 59 \\
\hline \multicolumn{5}{|c|}{ POLYMER-BASED NASAL MUCOADHESIVE NANOCARRIERS } \\
\hline Rivastigmine & Chitosan & Chitosan NPs & Alzheimer's disease & 60 \\
\hline Ropinirole $\mathrm{HCl}$ & Chitosan & Chitosan NPs & Parkinson's disease & 61 \\
\hline Bromocriptine & Chitosan & Chitosan NPs & Parkinson's disease & 62 \\
\hline Tetanus toxoid (TT) & Chitosan & Chitosan NPs & Immunization against TT antigen & 63 \\
\hline Ovalbumin & $\mathrm{N}, \mathrm{N}, \mathrm{N}$-trimethyl chitosan (TMC) & TMC NPs & NM & 64 \\
\hline Leucine-enkephalin & TMC & TMC NPs & Acute and chronic pain & 65 \\
\hline Ropinirole $\mathrm{HCl}$ & TMC & TMC NPs & Parkinson's disease & 66 \\
\hline Ropinirole $\mathrm{HCl}$ & TMC & TMC-DS PECs & Parkinson's disease & 67 \\
\hline Insulin & Starch & Starch NPs & Hyperglycemia /Diabetes & 68 \\
\hline HBsAg & Chitosan, Glycol chitosan & PLGA NPs & Hepatitis B & 69 \\
\hline Chlorpromazine & Chitosan & PLGA NPs & Schizophrenia & 70 \\
\hline Venlafaxine & Alginate & Alginate NPs & Depression & 71 \\
\hline \multicolumn{5}{|c|}{ PROTEIN-BASED NASAL MUCOADHESIVE NANOCARRIERS } \\
\hline Tacrine & $H P-\beta-C D, S B E-\beta-C D$ & Albumin NPs & Alzheimer's disease & 72 \\
\hline
\end{tabular}




\section{Lipid-based nasal mucoadhesive nanocarriers Solid lipid nanoparticles (SLNs)}

The solid lipid-based nanoparticles (SLNs) have been reported to enhance the absorption of intranasally administered drug molecules through modification of the surface of SLNs using mucoadhesive polymer (chitosan) $)^{43}$ or by incorporating the preformed SLNs into Pluronic-based gel. ${ }^{44}$ Recently, Rassu et al. (2017) proposed a nasal drug delivery system based on mucoadhesive SLNs to exploit both the olfactory and trigeminal nerve pathways in order to promote the transport of siRNA into the central nervous system (CNS). A modified solvent emulsification evaporation method based on a $\mathrm{w} / \mathrm{o} / \mathrm{w}$ double-emulsion technique was employed to prepare SLNs and further surface-modified using chitosan in order to obtain an efficient and optimal nose-to-brain transport of BACE1 siRNA, potentially useful in the treatment of Alzheimer's disease. ${ }^{43}$

\section{Nanostructured lipid carriers (NLCs)}

Nanostructured lipid carriers (NLCs), comprising a mixture of solid and liquid lipids, has been utilized for the effective delivery of variety of drug molecules. However, there are but a single report demonstrating the nose to brain drug delivery using surface engineered NLCs. In an investigation by Devkar et al. (2014), ondansetron hydrochloride (OND) loaded NLCs were prepared by using high pressure homogenization technique and further surface modified using Delonix regia gum, a naturally occurring mucoadhesive polymer. The increase in particle size and shifting of zeta potential values towards positive side indicated the surface modification of the NLCs by the mucoadhesive polymer. The mucoadhesive strength imparted to the NLCs resulted in increased residence time of the formulation on the nasal mucosal membrane ensuring rapid and high nasal absorption of OND. The higher values of drug targeting efficiencies confirmed the efficiency of OND-loaded NLCs in nose to brain drug delivery. ${ }^{45}$

\section{Polymer-lipid hybrid nanoparticles (PLNs)}

In 2013, our group (Pardeshi et al. 2013b) reported the fabrication and evaluation of novel surface modified polymer-lipid hybrid nanoparticles (PLN) as robust carriers for intranasal delivery of ropinirole hydrochloride $(\mathrm{ROPI} \mathrm{HCl})$, an anti-parkinsonian agent. Sustained release, avoidance of hepatic first pass metabolism and improved therapeutic efficacy, through enhanced retention of formulation in nasal cavity are the major objectives of the proposed PLN formulation. PLN were fabricated by emulsification-solvent diffusion technique. We employed trimyristine as a solid lipid and HPMC K15M as a mucoadhesive polymer. In conclusions, the PLN was found to be safe and robust nanocarrier for the intranasal delivery of hydrophilic ROPI $\mathrm{HCl}$, especially in treatment of Parkinson's disease. ${ }^{46}$

\section{Liposomes}

The nasal route of administration holds a great promise in the systemic delivery of small molecules, peptides like insulin and genetic immunization (DNA vaccination) due to particular organization of the nasal mucosa. ${ }^{47-49}$ The nasal mucosae is the first site of contact with inhaled macromolecules and the nasal associated lymphoid tissue (NALT) at the base of the nasal cavity (Waldeyer's ring in humans) is important in the defense of mucosal surfaces. Additionally, the nasal epithelium is leaky and there are underlying blood vessels, cervical lymph nodes and lymphoid cells to which the macromolecule may have direct access if it can be adequately transported across the epithelium. Hence, nasal mucosal immunization offers significant advantages in terms delivery, safety and efficacy, in comparison to traditional systemic delivery of vaccines. ${ }^{49}$

Khatri et al. (2008) investigated the potential utility of glycol chitosan-coated liposomes carrying plasmid DNA as nasal vaccine delivery vehicle, in mice, for producing viral specific humoral mucosal and cellular immune response. Liposomes were prepared by dehydrationrehydration method and subsequently coated with glycol chitosan by simple incubation method. Upon intranasal administration, glycol chitosan-coated liposomes elicited humoral mucosal and cellular immune responses that were significant as compared to naked DNA justifying the potential advantage of mucosal vaccination in the production of local antibodies at the sites where pathogens enters the body. ${ }^{49}$

\section{Microemulsions (MEs) and nanoemulsions (NEs)}

The only scientifically important difference among microemulsions (MEs) and nanoemulsions (NEs) is that the MEs are the equilibrium systems which are thermodynamically stable while the NEs are nonequilibrium systems which are kinetically stable. Diverse studies have been reported on the development of MEs and NEs for brain targeting after intranasal administration. ${ }^{50-59}$

Porecha et al. (2009) prepared the mucoadhesive MEs loaded with diazepam, Lorazepam and alprazolam and proposed for the treatment of insomnia. MEs were prepared by titration method. The fabricated MEs were evaluated for sleep induction studies in male albino rats to assess their role in effective relief of insomnia 
patients. It was observed, from their findings, that the intranasal mucoadhesive MEs had shown the fastest onset of sleep ( $<9 \mathrm{~min})$ and longest duration of sleep as well in rats. ${ }^{54}$

Sood et al. (2014) fabricated the curcumin-loaded chitosan-coated mucoadhesive NEs using spontaneous nanoemulsification method and intended for intranasal administration. The alteration of the zeta potential values indicated the successful coating of chitosan on the NE globules. The developed formulation was found to be non-toxic and safe for intranasal administration as demonstrated by the in vitro cytotoxicity (on SK-N-SH cells, a human neuroblastoma cell lines) and nasal ciliotoxicity studies. In addition, the nasal mucoadhesive NEs had shown enhanced flux and permeability coefficient across sheep nasal mucosal membrane compared to uncoated NEs and bulk drug solution, indicating the suitability of mucoadhesive NEs for intranasal delivery of poorly soluble curcumin..$^{58}$

\section{Polymer-based nasal mucoadhesive nanocarriers}

There is a numerous literature available demonstrating the findings on the polymer-based mucoadhesive nanocarriers developed so far for intranasal administration. The prime objective being to enhance the retention time of nanocarrier formulations on the nasal mucosa, in order to improve the nasal mucosal absorption and permeability characteristics. These nanocarriers are extensively investigated for the delivery of variety of therapeutic agents' vi\% small drug molecules, proteins, peptides, vaccines and other macromolecules. The polymer-based mucoadhesive nanocarriers are mainly made of either mucoadhesive polymers like chitosan, ${ }^{60-63}$ TMC, ${ }^{64-67}$ or made from non-mucoadhesive polymers like starch, ${ }^{68}$ PLGA, ${ }^{69,70}$ alginate, ${ }^{71}$ etc. but further surface-modified with mucoadhesive polymers.

Recently, our group (Kulkarni et al. 2016) fabricated the new self-assembled polyelectrolyte nanocomplex based on two mucoadhesive polymers vir. N,N,N-trimethyl chitosan (TMC) and dextran sulfate (DS), the nanocomplex formulation being referred to as TMC-DS PECs. The PECs were prepared by using ionic interactions between charged functional groups of both the participating mucoadhesive polymers at different $\mathrm{pH}$

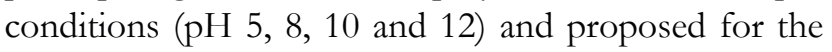
intranasal delivery of ROPI HCl. It has been observed, from the permeation experiments, that the PECs prepared at $\mathrm{pH} 10$ shows high permeability of ROPI $\mathrm{HCl}$ across sheep nasal mucosa via paracellular route of drug transport (that takes place between the adjacent epithelial cells through hydrophilic pores and the tight junctions between the cells). Again, the in vitro muco- adhesion potential of prepared PECs was assessed by mucus glycoprotein assay. The mucin binding efficiency (MBE) of TMC-DS PEC prepared at $\mathrm{pH} 10$ found to be rated as good mucoadhesive strength, based on a rating criteria for mucin adsorption on a mucoadhesion-scale from poor $(\mathrm{MBE}<50 \%)$ to excellent $(\mathrm{MBE}>75 \%)$. The high mucoadhesive strength is due to a highly specific, concentration-dependent adsorption with strong electrostatic interactions between positively charged trimethyl amino groups of TMC and negatively charged sialic and sulfonic acid moieties in mucin. It was proposed that the higher adsorption of mucin on PEC particles would result in better penetration of PEC particles in mucosal layer. At alkaline $\mathrm{pH}$ conditions ( $\mathrm{pH}$ 10), the participant polymer chains (TMC and DS) are sufficiently close to form more stable PECs. Authors concluded that the further in vivo brain pharmacokinetics, scintigraphic/microscopical imaging and stability studies need to be addressed appropriately in order to validate the biofate of TMCDS PECs as novel nose to brain drug delivery carriers and further, their scale-up. ${ }^{67}$

\section{Protein-based nasal mucoadhesive nanocarriers}

The only report on protein-based nasal mucoadhesive nanocarriers for drug delivery application is given by Luppi et al. (2011). In their investigations, the researchers employed coacervation method for the preparation of bovine serum albumin (BSA) NPs. The nanoparticles were loaded with tacrine, the first acetylcholinestearse inhibitor licensed for the treatment of Alzheimer's disease. The effect of three difference cyclodextrins vi\%: beta cyclodextrin $(\beta-C D)$, hydroxypropyl beta cyclodextrin $(\mathrm{HP}-\beta-\mathrm{CD})$ and sulphobutylether beta cyclodextrin $(\mathrm{SBE}-\beta-\mathrm{CD})$ on the lading efficiency, mucoadhesion potential, drug release and permeation characteristics of tacrine was examined. From the results, it has been observed the inclusion of different $\mathrm{CDs}$ into the albumin NPs has modulated the permeability of tacrine, more specifically the albumin NPs carrying HP- $\beta-C D$ had shown maximum permeability across the nasal mucosa. ${ }^{72}$

\section{Regulatory aspects and clinical status}

Interest in the use of mucoadhesive polymers in the formulation of nanopharmaceuticals is not new however, it still does not appear in much marketed products. Despite of the long list of nanopharmaceuticals approved by FDA (Food and Drug Administration) for use in clinic by numerous routes of administration but mucoadhesive nanopharmaceuticals delivered via intranasal route are still under investigation into the laboratories. The lack of sufficient safety data and valid outcomes, very few of the mucoadhesive NP formula- 
tions have found a way to the commercial marker. In addition, there are but few patents on the mucoadhesive NPs for intranasal administration. ${ }^{73-76}$ However, none of them have translated to the clinical phase, even after being investigated extensively in the laboratory. For instance, about 22 clinical trials are going-on on the nanopharmaceuticals by various routes of administration and the excellent preclinical outcomes have energized the researchers for the further clinical advancements, but the researchers are still waiting for the encouraging results in human subjects.

\section{Into the future}

At present, the nasal mucoadhesive nanoparticulate formulation have proved their wide applicability in various fields. On laboratory scale, the excellent research outocomes have been reported with significant early preclinical success, alarming the pharmaceutical industries to take over the challenge of successful commercialization of such formulations. However, it is very important to make a note on the crucial aspect that the selection of ideal mucoadhesive polymer, ideal nanocarrier formulation and the ideal drug candidate for a particular mucoadhesive nanocarrier formulation is a task of extreme significance in order to develop a safe and stable dosage form successfully. Again, the toxicity experiments on the polymer- or lipid-based nanocarriers carrying therapeutic payloads must be performed so as to provide additional evidences on the risks associated with the development of such nanocarrier formulations.

To look into the future, the upcoming developments in the mucoadhesive nanocarrier formulations for intranasal administration need to be extensively explored with respect to increasing performance, improving the absorption or permeability characteristics and reducing the toxicity issues.

\section{CONCLUSION}

To be called as a successful drug delivery system, the formulation must offer commercial applicability to the pharmaceutical industries for large scale production. Among all developed novel drug delivery systems, the multiparticulate drug delivery systems have broad prospects in the pharmaceutical field. The most promising outcomes (greater therapeutic efficacy and reduced dosing frequency) received from the nanocarrier formulations have attracted numerous research groups from various disciplines to work over. Among the much vastly investigated drug delivery systems, the mucoadhesive nanoparticulate dosage forms gained much attention today in formulation development due to their inherited advantages like prolonged residence time, uniform distribution at the target site, rapid and more reproducible drug absorption.

The nasal mucoadhesive nanoparticulate drug delivery systems have already proved their potential in the efficient delivery of pharmaceuticals, on laboratory scale. However, to bring such nanocarrier formulations to the commercial market, extensive clinical research is needed. If the encouraging results are obtained, the day is not so far when we see the nasal mucoadhesive nanoformulations on the pharmacist's shelves.

\section{ACKNOWLEDGEMENT}

The authors are grateful to the Management and Principal of R. C. Patel Institute of Pharmaceutical Education and Research, Shirpur (Maharashtra) for providing necessary facilities to carry out the research work under this project.

\section{Funding information}

The authors received no funding for this project.

\section{Statement of Human and Animal Rights}

This article does not contain any studies with human or animal subjects performed by any of the authors.

\section{CONFLICT OF INTEREST}

The authors declare no conflict of interest.

\section{ABBREVIATIONS}

NPs: Nanoparticles; IN: Intranasal; NIH: National Institute of Health; FDA: Food and Drug Administration; EMA: European Medicines Agency; nm: Nanometers; DOX: Doxorubicin; PEG: Polyethylene glycol; GI: Gastrointestinal; Mw: Molecular weight; MCC: Mucociliary clearance mechanism; SLNs: Solid lipid nanoparticles; CNS: Central nervous system; NLCs: Nanostructured lipid carriers; OND: Ondansetron hydrochloride; PLN: Polymer-lipid hybrid nanoparticles; ROPI HCl: Ropinirole hydrochloride; HPMC: Hydroxypropyl methyl cellulose; NALT: Nasal associated lymphoid tissue; MEs: Microemulsions; NEs: Nanoemulsions; TMC: N,N,N-trimethyl chitosan; DS: Dextran sulfate; PEC: Polyelectrolyte complex; MBE: Mucin binding efficiency; BSA: Bovine serum albumin; CD: Cyclodextrin; SBE: Sulfobutylether; NLC: Nanostructured lipid carriers; PLN: Polymer-lipid hybrid nanoparticles; CHT: Chitosan; HCl: Hydrochloride; HSV: Herpes simplex virus; PVP: Poly-N-Vinyl-2-Pyr- 
rolidone; HBsAg: Hepatitis B surface antigen; PECs: Polyelectrolyte complex; HP- $\beta-\mathbf{C D}$ : Hydroxypropyl beta cyclodextrin; SBE- $\beta$-CD: Sulphobutylether beta cyclodextrin; NM: Not mentioned.

\section{REFERENCES}

1. Patra CR, Bhattacharya R, Mukhopadyay D, Mukherjee P. Fabrication of gold nanoparticles for targeted therapy in pancreatic cancer. Adv Drug Deliv Rev. 2010;62(3):346-61.

2. Webster TJ. Nanomedicine: What's in a definition?. Int $\mathrm{J}$ Nanomed 2006;1:115-6.

3. National Institutes of Health. 2006. National Institute of Health Roadmap for Medical Research: Nanomedicine. Available online at: http://nihroadmap.nih. gov/nanomedicine/.

4. National Nanotechnology Initiative. Available online at http://www.nano.gov

5. Pardeshi C, Rajput P, Belgamwar V, Tekade A, Patil G, Chaudhary K, et al. Solid lipid based nanocarriers: an overview. Acta Pharm. 2012;62(4):433-72.

6. Pardeshi CV, Rajput PV, Belgamwar VS, Tekade AR. Formulation, optimization and evaluation of spray-dried mucoadhesive microspheres as intranasal carriers for valsartan. J Microencapsul. 2012;29(2):103-14.

7. Available online at http://www.scientificamerican.com/article.cfm?id=sizeshape-matter-nanotech-drug.

8. Patel S, Patel B, Patel Z, Pardeshi C. Nanocarriers as novel nose-to-brain targeted drug delivery platforms. Indian Journal of Novel Drug Delivery. 2012;4:243-51.

9. Mistry A, Stolnik S, Illum L. Nanoparticles for direct nose-to-brain delivery of drugs. Int J Pharm. 2009;379(1):146-57.

10. Ugwoke MI, Agu RU, Verbeke N, Kinget R. Nasal mucoadhesive drug delivery: Background, applications, trends and future perspectives. Adv Drug Deliv Rev. 2005;57(11):1640-65.

11. Pardeshi CV, Belgamwar VS. Direct nose to brain drug delivery via integrated nerve pathways bypassing the blood-brain barrier: an excellent platform for brain targeting. Expert Opin Drug Deliv. 2013;10(7):957-72.

12. Pardeshi CV, Vanjari YH, Kulkarni AD. Novel nasal devices for the efficient drug delivery: a systemic review. Indian Journal of Novel Drug Delivery. 2015;7:1-9.

13. Carvalho FC, Bruschi ML, Evangelista RC, Gremiao MPD. Mucoadhesive drug delivery system. Braz J Pharm Sci. 2010;46(1):1-7.

14. Chien YW. Novel Drug Delivery Systems, (2 ${ }^{\text {nd }}$ Ed. $)$, Marcel Decker Inc, New York. 1992;1-42.

15. Longer MA, Robinson JR. Fundamental aspects of bioadhesion. Pharmaceutics International. 1986;7(5):114-7.

16. Thirawong N, Kennedy RA, SriamornsakRA. Viscometric study of pectin-mucin interaction and its mucoadhesive bond strength. Carbohydr Polym 2008;71(2):170-9.

17. Ghanghoria R, Kesharwani P, Agashe HB, Jain NK. Transdermal delivery of cyclodextrin-solubilized curcumin. Drug Deliv TransI Res. 2013;3(3):272-85.

18. Kaur IP, Kakkar V, Deol PK, Yadav M, Singh M, Sharma I. Issues and concerns in nanotech product development and its commercialization. J Control Release. 2014;193:51-62.

19. Available online at http://www.nano.gov/nanotech-101/what/definition

20. Bawa R. Nanopharmaceuticals. Eur J Nanomed. 2010;3:34-9.

21. Available online at: https://www.bccresearch.com/marketresearch/ healthcare/nanotechnology-medical-applications-market-hlc069c.html BCC Research, Nanotechnology in medical applications: The Global Market. 2015.

22. Ragelle H, Danhier F, Preat V, Langer R, Anderson DG. Nanoparticle-based drug delivery systems: a commercial and regulatory outlook as the field matures. Expert Opin Drug Deliv 2017;14(7):851-64.

23. Mansour HM, Park CW, Bawa R. Design and development of approved nanopharmaceuticals products. Handbook of Clinical Nanomedicine-From Bench to Bedside, Pan Stanford Publishing Pvt. Ltd. Singapore. 2015;1-33.

24. Bernkop-Schnurch A. Mucoadhesive polymers: strategies, achievements and future challenges. Adv Drug Deliv Rev. 2005;57(11):1553-5.
25. Mansuri S, Kesharwani P, Jain K, Tekade RK, Jain NK. Mucoadhesion: A promising approach in drug delivery system. React Funct Polym. 2016;100:151-72.

26. Mathiowitz E, Chickering III DE. Bioadhesive Drug Delivery Systems: Fundamentals, novel approaches and development. Marcel Dekker, New York. 1999;1-10.

27. Andrews GP, Laverty TP, Jones DS. Mucoadhesive polymeri platform for controlled drug delivery. Eur J Pharm Biopharm. 2009;71(3):505-18.

28. Asane GS, Nirmal SA, Rasal KB, Naik AA, Mahadik MS, Rao YM. Polymers for mucoadhesive drug delivery system: a current status. Drug Dev Ind Pharm. 2008;34(11):1246-66.

29. Kumar K, Dhawan N, Sharma H, Vaidya S, Vaidya B. Bioadhesive polymers: Novel tool for drug delivery. Artif Cells Nanomed Biotechnol. 2014;42(4):274-83.

30. Sudhakar Y, Kuotsu K, Bandyopadhyay AK. Buccal bioadhesive drug delivery-a Promising option for orally less efficient drugs. J Control Release. 2006;114(1):15-40.

31. Pardeshi CV, Rajput PV, Belgamwar VS, Tekade AR, Surana SJ. Novel surface modified solid lipid nanoparticles as intranasal carriers for ropinirole hydrochloride: application of factorial design approach. Drug Deliv. 2013;20(1):47-56.

32. Kulkarni AD, Bari DB, Surana SJ, Pardeshi CV. In vitro, ex vivo and in vivo performance of chitosan-based spray-dried nasal mucoadhesive microspheres of diltiazem hydrochloride. J Drug Deliv Sci Technol. 2016;31:108-17.

33. Jadhav KR, Gambhire MN, Shaikh IM, Kadam VJ, Pisal SS. Nasal drug delivery system- factors affecting and applications. Curr Drug Ther. 2007;2(1):27-38.

34. Patel Z, Patel B, Patel S, Pardeshi CV. Nose to brain targeted drug delivery bypassing the blood-brain barrier: an overview. Drug Invention Today. 2012;4(12):610-5.

35. Kulkarni AD, Vanjari YH, Sancheti KH, Belgamwar VS, Surana SJ, Pardeshi CV. Nanotechnology-mediated nose to brain drug delivery for Parkinson's disease: a mini review. J Drug Target. 2015;23(9):775-88.

36. Available online at: www.britannica.com

37. Illum L. Nasal drug delivery-Possibilities, problems and solutions. J Control Release. 2003;87(1-3):187-98.

38. Illum L. Nasal clearance in health and disease. J Aerosol Med. 2006;19(1):92-9.

39. Merkus FW, Verhoef JC, Schipper NG, Marttin E. Nasal mucociliary clearance as a factor in nasal drug delivery. Adv Drug Deliv Rev. 1998;29(1-2):13-38.

40. Schipper NG, Verhoef JC, Merkus FW. The nasal mucociliary clearance: relevance to nasal drug delivery. Pharm Res. 1991;8(7):807-14.

41. Khanvilkar K, Donovan MD, Flanagan DR. Drug transfer through mucus. Adv Drug Deliv Rev. 2001;48(2-3):173-93.

42. Jones $\mathrm{N}$. The nose and paranasal sinuses physiology and anatomy. Adv Drug Deliv Rev. 2001;51(1-3):5-19.

43. Rassu G, Soddu E, Posadino AM, Pintus G, Sarmento B, Giunchedi P, Gavini E. Nose-to-brain delivery of BACE1 siRNA loaded in solid lipid nanoparticles for Alzheimer's therapy. Colloids Surf B Biointerfaces. 2017;152:296-301.

44. Khan MW, Ahsan MJ, Gupta SK. Carbamazepine loaded mucoadhesive solid lipid nanoparticle: Formulation and in-vitro characterization. Int J Adv Pharm Med Bioallied Sci. 2017;2017:1-9.

45. Devkar TB, Tekade AR, Khandelwal KR. Surface engineered nanostructured lipid carriers for efficient nose to brain delivery of ondansetron $\mathrm{HCl}$ using Delonix regia gum as a natural mucoadhesive polymer. Colloids Surf $B$ Biointerfaces. 2014;122:143-50.

46. Pardeshi CV, Belgamwar VS, Tekade AR, Surana SJ. Novel surface modified polymer-lipid hybrid nanoparticles as intranasal carriers for ropinirole hydrochloride: in vitro, ex vivo and in vivo pharmacodynamic evaluation. J Mater Sci Mater Med. 2013;24(9):2101-15.

47. Jain AK, Chalasani KB, Khar RK, Ahmed FJ, Diwan PV. Muco-adhesive multivesicular liposomes as an effective carrier for transmucosal insulin delivery. J Drug Target. 2007;15(6):417-27.

48. Alsarra IA, Hamed AY. Acyclovir liposomes for intranasal systemic delivery: development and pharmacokinetics evaluation. Drug Deliv. 2008;15(5):313-21.

49. Khatri K, Goyal AK, Gupta PN, Mishra N, Mehta A, Vyas SP. Surface modified liposomes for nasal delivery of DNA vaccine. Vaccine. 2008;26(18):2225-33. 
50. Vyas TK, Babbar AK, Sharma RK, Misra A. Intranasal mucoadhesive microemulsions of zolmitriptan: Preliminary studies on brain-targeting. J Drug Target 2005;13(5):317-24.

51. Vyas TK, Babbar AK, Sharma RK, Singh S, Misra A. Preliminary braintargeting studies on intranasal mucoadhesive microemulsions of Sumatriptan. AAPS PharmSciTech. 2006;7(1):E1-E9.

52. Vyas TK, Babbar AK, Sharma RK, Singh S, Misra A. Intranasal mucoadhesive microemulsions of clonazepam: preliminary studies on brain targeting. $J$ Pharm Sci. 2006;95(3):570-80.

53. Jogani VV, Shah PJ, Mishra P, Mishra AK, Misra AR. Intranasal mucoadhesive microemulsion of tacrine to improve brain targeting. Alzheimer Dis Assoc Disord. 2008;22(2):116-24.

54. Porecha S, Shah T, Jogani V, Naik S, Misra A. Microemulsion based intranasal delivery system for treatment of insomnia. Drug Deliv. 2009;16(3):128-34.

55. Kumar M, Misra A, Babbar AK, Mishra AK, Mishra P, Pathak K. Intranasal nanoemulsion based brain targeting drug delivery system of risperidone. Int J Pharm. 2008;358(1-2):285-91.

56. Kumar M, Pathak K. Formulation and characterization of nanoemulsion-based drug delivery system of risperidone. Drug Dev Ind Pharm. 2009;35(4):387-95.

57. Hosny KM, Banjar ZM. The formulation of a nasal nanoemulsion zaleplon in situ gel for the treatment of insomnia. Expert Opin Drug Deliv. 2013;10(8):1033-41.

58. Sood S, Jain K, Gowthamarajan K. Optimization of curcumin nanoemulsion for intranasal delivery using design of experiment and its toxicity assessment. Colloids Surf B Biointerfaces. 2014;113:330-7.

59. Mustafa G, Ahuja A, Al Rohaimi AH, Muslim S, Hassan AA, Baboota S, et al. Nano-ropinirole for the management of Parkinsonism: bloodbrain pharmacokinetics and carrier localization. Expert Reviews in Neurotherapeutics. 2015;15(6):695-710.

60. Fazil M, Md S, Haque S, Kumar M, Baboota S, Sahni JK, et al. Development and evaluation of rivastigmine loaded chitosan nanoparticles for brain targeting. Eur J Pharm Sci. 2012;47(1):6-15.

61. Jafarieh O, Md S, Ali M, Baboota S, Sahni JK, Kumari B, et al. Design, characterization and evaluation of intranasal delivery of ropiniroleloaded mucoadhesive nanoparticles for brain targeting. Drug Deliv. 2015;41(10):1674-81.

62. Md S, Khan RA, Mustafa G, Chuttani K, Baboota S, Sahni JK, et al. Bromocriptine loaded chitosan nanoparticles intended for direct nose to brain delivery: Pharmacodynamic, Pharmacokinetic and Scintigraphy study in mice model. Eur J Pharm Sci. 2013;48(3):393-405.

63. Vila A, Sanchez A, Janes K, Behrens I, Kissel T, Jato JLV, et al. Low molecular weight chitosan nanoparticles as new carriers for nasal vaccine delivery in mice. Eur J Pharm Sci. 2004;57(1):123-31.
64. Amidi M, Romeijn SG, Borchard G, Junginger HE, Hennink WE, Jiskoot W. Preparation and characterization of protein-loaded N-trimethyl chitosan nanoparticles as nasal delivery system. J Control Release. 2006;111(1-2): 107-16.

65. Kumar M, Pandey RS, Patra KC, Jain SK, Soni ML, Dangi JS, et al. Evaluation of neuropeptide loaded trimethyl chitosan nanoparticles for nose to brain delivery. Int J Biol Macromol. 2013;61:189-95.

66. Pardeshi CV, Belgamwar VS. Controlled synthesis of N,N,N-trimethyl chitosan for modulated bioadhesion and nasal membrane permeability. Int $\mathrm{J}$ Biol Macromol. 2016;82:933-44.

67. Kulkarni AD, Vanjari YH, Sancheti KH, Patel HM, Belgamwar VS, Surana SJ, et al. New nasal nanocomplex self-assembled from charged biomacromolecules: N,N,N-trimethyl chitosan and dextran sulfate. Int J Biol Macromol. 2016;88:476-90.

68. Jain AK, Khar RK, Ahmed FJ, Diwan PV. Effective insulin delivery using starch nanoparticles as a potential trans-nasal mucoadhesive carrier. Eur $\mathrm{J}$ Pharm Biopharm. 2008;69(2):426-35.

69. Pawar D, Mangal S, Goswami R, Jaganathan KS. Development and characterization of surface modified PLGA nanoparticles for nasal vaccine delivery: Effect of mucoadhesive coating on antigen uptake and immune adjuvant activity. Eur J Pharm Biopharm. 2013;85(3):550-9.

70. Chalikwar SS, Mene BS, Pardeshi CV, Belgamwar VS, Surana SJ. Selfassembled, chitosan grafted PLGA nanoparticles for intranasal delivery: design, development and ex vivo characterization. Polym Plast Technol Eng. 2013;52(4):368-80.

71. Haque S, Md S, Sahni JK, Ali J, Baboota S. Development and evaluation of brain targeted intranasal alginate nanoparticles for treatment of depression. J Psychiatr Res. 2014;48(1):1-12.

72. Luppi B, Bigucci F, Corace G, Delucca A, Cerchiara T, Sorrenti M, et al. Albumin nanoparticles carrying cyclodextrins for nasal delivery of the antiAlzheimer drug tacrine. Eur J Pharm Sci. 2011;44(4):559-65.

73. Misra AN, Vyas TK. Sedatives loaded intranasal nasoadhesive microemulsions for brain targeted delivery in insomnia. Indian Patent. 2004. 1124/MUM/2004.

74. Misra AN, Vyas TK. Drugs loaded intranasal nasoadhesive microemulsions for brain targeted delivery in migraine. Indian Patent 2004. 1125/MUM/2004.

75. Pardeshi CV, Belgamwar VS. Novel neuronanoemulsion. Indian Patent. 2015. 666/MUM/2015.

76. Pardeshi CV, Belgamwar VS. Novel nanocarrier composition for brain targeting. 2015. 667/MUM/2015.

\section{SUMMARY}

- The multiparticulate formulations possess high commercial scalability among all novel drug delivery systems, now-a-days, in pharmaceutical field.

- The most promising outcomes (greater therapeutic efficacy and reduced dosing frequency) received from the nanocarrier formulations have attracted numerous research groups from various disciplines to work over.

- The lack of sufficient safety data and valid outcomes, very few of the mucoadhesive NP formulations have found a way to the commercial marker. In addition, there are but few patents on the mucoadhesive NPs for intranasal administration.

- The nasal mucoadhesive nanoparticulate drug delivery systems have already proved their potential in the efficient delivery of pharmaceuticals, on laboratory scale. However, to bring such nanocarrier formulations to the commercial market, extensive clinical research is needed. If the encouraging results are obtained, the day is not so far when we see the nasal mucoadhesive nanoformulations on the pharmacist's shelves. 


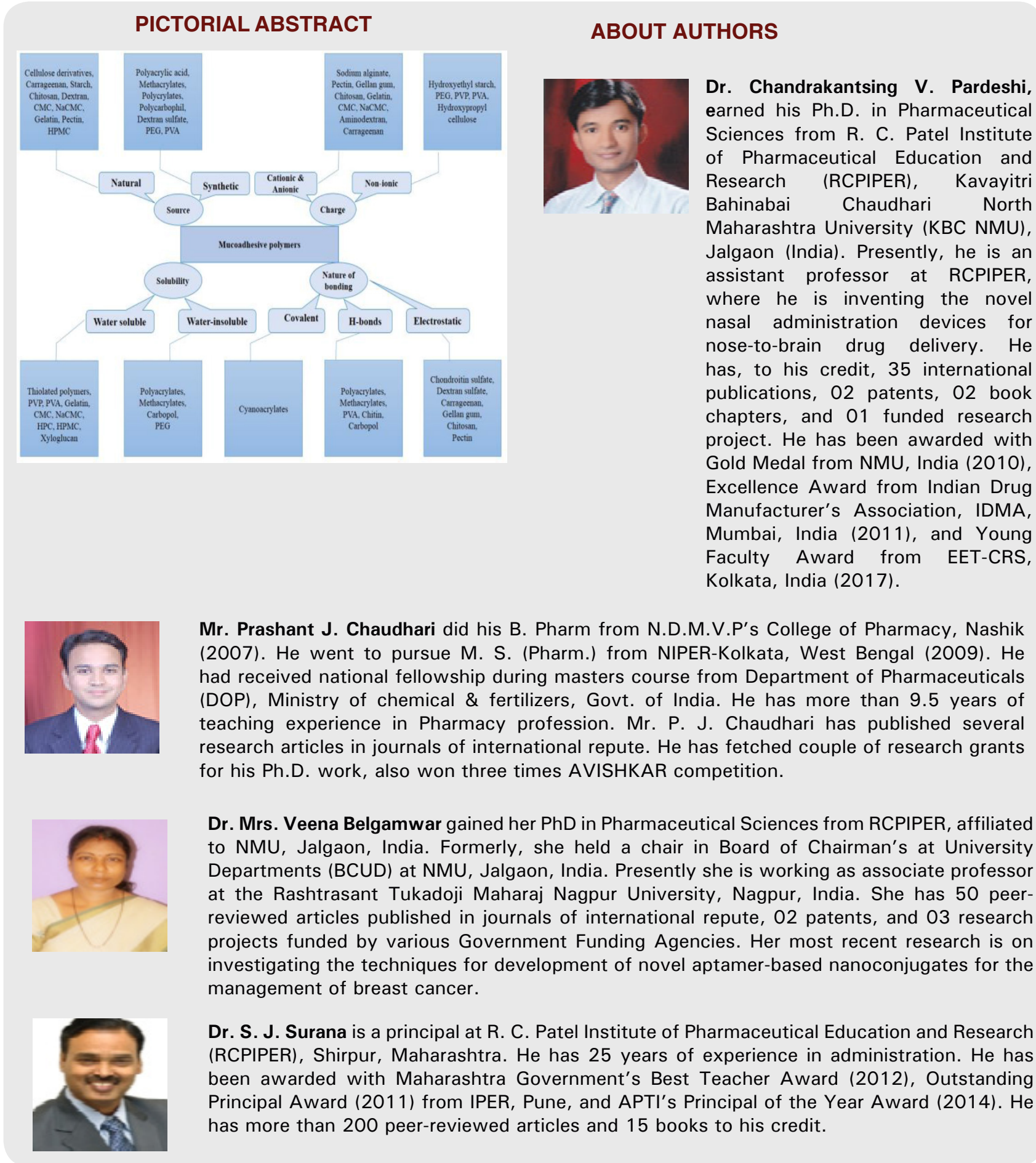

Cite this article: Pardeshi CV, Kulkarni AD, Sonwane RO, Belgamwar VS, Chaudhari PJ, Surana SJ. Mucoadhesive Nanoparticles: A Roadmap to Encounter the Challenge of Rapid Nasal Mucociliary Clearance. Indian J of Pharmaceutical Education and Research. 2019;53(2S):s17-s27. 